\title{
Avaliação de diferentes modelos de secagem para liofilização de mangabas maduras com diferentes diâmetros através de indicadores de desempenho
}

Evaluation of different drying models for freeze drying of ripe mangabas with different diameters through of performance indicators

\author{
A. M. Oliveira Júnior ${ }^{1 *}$; D. S. C. Soares ${ }^{1}$; J. T. S. Santos ${ }^{1}$; T. P. Nunes ${ }^{1}$ \\ ${ }^{1}$ Departamento de Tecnologia de Alimentos, Universidade Federal de Sergipe, 49100-000, São Cristóvão-Sergipe, \\ Brasil \\ *amartins.junior@gmail.com \\ (Recebido em 15 de março de 2016; aceito em 15 de abril de 2016)
}

\begin{abstract}
Os modelos matemáticos de secagem são empregados com o intuito de caracterização do processo ao estimar parâmetros importantes para o mesmo. A mangaba (Hancornia speciosa Gomes) é uma fruta de clima tropical com elevada perecibilidade, este aspecto propõe o uso de técnicas alternativas para promover o aumento da vida útil, dentre está a liofilização. Diante disto, objetivou-se analisar diversos modelos matemáticos (Page, Henderson e Pabis, Lewis e Midilli) para avaliar aquele que melhor se adequa as condições do processo, por meio de índices de desempenho associados a cada modelo: fatores de precisão $\left(\mathrm{A}_{\mathrm{f}}\right)$ e de viés $\left(\mathrm{B}_{\mathrm{f}}\right)$, raiz do erro quadrático médio (REQM) e erro padrão de predição $(\% \mathrm{SEP})$. As frutas foram congeladas à temperatura de $-20{ }^{\circ} \mathrm{C}$ em freezer convencional, seguida da liofilização, em liofilizador a $-50{ }^{\circ} \mathrm{C}$ e vácuo parcial de $38 \mu \mathrm{mHg}$ durante $1380 \mathrm{~min}$. Mediante os resultados, observa-se que o modelo de Page se mostrou mais preciso independentemente do diâmetro, com $\mathrm{A}_{\mathrm{f}}$ : 1,9198, $\mathrm{B}_{\mathrm{f}}: 1,8446$, REQM: 0,0275 e \%SEP: 5,6298 e $\mathrm{A}_{\mathrm{f}}: 1,7334 \mathrm{~B}_{\mathrm{f}}:$ 1,6735; REQM: 0,0221 e \%SEP: 3,5545 para as mangabas pequenas e grandes, respectivamente. Diante disto, nota-se que a melhor correlação entre os dados experimentais e estimados é dada pelo Modelo de Page.

Palavras-chave: Secagem, modelagem, Hancornia speciosa Gomes
\end{abstract}

Drying mathematic models are used to characterize the drying process by estimating important parameters to the processes. Mangaba (Hancornia speciosa Gomes) is a tropical climate fruit with high perishability. Due to this fact, it is necessary alternative processes to increase its shelf life, such as freeze-drying. The objective of this work was to analyze several models in order to evaluate the best model according to the process conditions through the indices of the performance of models which are: accuracy factor $\left(\mathrm{A}_{\mathrm{f}}\right)$, bias factor $\left(\mathrm{B}_{\mathrm{f}}\right)$, root mean square error (RMSE) and standard error of prediction (\%SEP). The fruits were frozen at $-20{ }^{\circ} \mathrm{C}$ in conventional freezer, and then freeze-dried at $-50{ }^{\circ} \mathrm{C}$ and $38 \mu \mathrm{mHg}$ for $1380 \mathrm{~min}$. The results showed that the model of Page was precise independent of the diameter, with: $\mathrm{A}_{\mathrm{f}}$ : 1.9198, $\mathrm{B}_{\mathrm{f}}: 1.8446$, RMSE: 0.0275 e \%SEP: 5.6298 and $\mathrm{A}_{\mathrm{f}}: 1.7334 \mathrm{~B}_{\mathrm{f}}$ : 1.6735 ; RMSE: $0.0221 \mathrm{e} \% \mathrm{SEP}: 3.5545$ for the small and large mangabas, respectively. It was noted that the model of Page resulted in a better correlation between the experimental and estimated data.

Keywords: Drying, models, Hancornia speciosa Gomes

\section{INTRODUÇÃO}

O nordeste brasileiro tem uma diversidade natural de frutas e muitas delas são consideradas exóticas devido aos seus aromas e sabores diferentes. Devido a estas características peculiares diversas pesquisas são concentradas nestas frutas, assim como novos consumidores são atraídos mundialmente. As frutas tropicais tem um alto potencial para o processamento de sucos, sobremesas e outros produtos processados [1].

Dentre as frutas tropicais, encontra-se a Hancornia speciosa Gomes, popularmente conhecida como mangaba. A mangaba é uma fruta rica em ácido ascórbico e aromas, além disso, é muito utilizada para a fabricação de sorvetes, sucos e vinagres no nordeste do Brasil. Quando madura, a mangaba é bastante perecível o que dificulta a exportação desta matéria prima na sua forma in 054210 - 1 
natura, sendo necessários processos que possibilitem um aumento na vida de prateleira do produto [2].

Como uma alternativa para estender a vida útil e facilitar a comercialização, a secagem de frutas tropicais pode ser utilizada com excelência. Devido à redução da atividade de água e consequentemente, a prevenção de contaminação microbiológica e de reações bioquímicas indesejáveis, a secagem permite que um produto perecível se torne um produto estável para a comercialização e consumo. Além disso, a secagem reduz as perdas pós-colheita e pode agregar valor aos produtos [3]. Devido às propriedades das frutas tropicais, a liofilização tem sido amplamente utilizada, pois é um processo onde ocorre a preservação e retenção de atributos da fruta, como por exemplo, aparência, nutrientes, cor e sabor [4].

O estudo sobre liofilização é de suma importância quando se trata de materiais para os quais as informações sobre o comportamento cinético de secagem são escassas, possibilitando o estudo da influência das variáveis do processo sobre a transferência de massa. Neste sentido, o estudo da cinética de secagem é de fundamental importância para a predição das equações de taxa de secagem utilizadas na modelagem dos fenômenos de transferência na secagem [5]. A disposição de modelos que simulem as curvas de secagem sob condições diferentes é necessário [6]. Com isto modelos matemáticos que são classificados em teóricos, semiteóricos e empíricos tem sido propostos para descrever os processos de secagens [7]. Os modelos semiteóricos tem sido amplamente utilizados devido a sua facilidade de uso, dentre os modelos que são comumente usados em alimentos incluem Page, Difusivo, Henderson e Pabis, Lewis, Midilli, dentre outros [8].

O presente trabalho tem como objetivo avaliar modelos semiteóricos para a liofilização de mangabas madura em dois diâmetros diferentes, por meio de indicadores de desempenho relacionados com cada modelo.

\section{MATERIAL E MÉTODOS}

O trabalho foi conduzido no Laboratório de Processamento de Origem Vegetal (LPOV), localizado no Departamento de Tecnologia de Alimentos (DTA), na Universidade Federal de Sergipe (UFS).

\subsection{Matéria-prima}

As mangabas no estágio de maturação maduro foram adquiridas na praia do Abaís, localizada na cidade Estância-SE e transportados para o laboratório sob resfriamento em baldes de gelo. As frutas foram selecionadas manualmente com a observação da cor da casca e textura. Em seguida, o material selecionado foi sanitizado em solução de cloro ativo a $200 \mathrm{ppm}$ por 10 minutos e posteriormente foi realizada a lavagem dos frutos em água potável corrente. Logo após a sanitização, os frutos foram congelados à temperatura de $-20{ }^{\circ} \mathrm{C}$ em freezer convencional para uma posterior liofilização.

\subsection{Liofilização}

A liofilização foi realizada com o fruto in natura com dois diferentes diâmetros. Para a liofilização, foi utilizado liofilizador da marca Liotop modelo L-108, a $-50{ }^{\circ} \mathrm{C}$ e vácuo parcial de $38 \mu \mathrm{mHg}$ durante $1380 \mathrm{~min}$. Durante este período foram feitas pesagens em intervalos de 30,60 e 180 min. Após o fim do processo, as amostras foram armazenadas em embalagens de polietileno laminadas.

\subsection{Modelagem Matemática}

A cinética de secagem das mangabas in natura com diferentes diâmetros foi realizada por meio de modelos semiteóricos (modelo de Page, Midilli, Henderson e Pabis e Lewis). A modelagem matemática foi avaliada por meio dos indicadores de desempenho: RMSE, \%SEP, $\mathrm{A}_{\mathrm{f}}$ e $\mathrm{B}_{\mathrm{f}}$.

Para fins de modelagem matemática, determinou-se a razão de umidade dos dados experimentais, por meio da Equação 1.

$$
R U=\frac{\left(U(t)-U_{e q}\right)}{\left(U_{i}-U_{e q}\right)}
$$

Onde: 
Ru corresponde a razão de umidade;

$\mathrm{U}(\mathrm{t})$ consiste na umidade no tempo t, em base seca;

$\mathrm{U}_{\text {eq }}$ a umidade no equilíbrio, em base seca e;

$\mathrm{U}_{\mathrm{i}}$ é a umidade inicial, em base seca.

Os dados experimentais da razão de umidade foram ajustados para os modelos descritos na Tabela 1. Para isto foi utilizado o software STATISTICA® versão 7.0 para Windows, no qual foi realizada uma análise de regressão não linear pelo método Levenberg-Marquardt com o método dos mínimos quadrados como função de perda.

A definição do modelo mais apropriado foi feita pelos índices de desempenho explícitos na Tabela 2, seus resultados foram calculados no software Excel® versão 2007 para Windows.

Tabela 1: Modelos matemáticos de secagem

\begin{tabular}{cc}
\hline Modelos Matemáticos & Equações \\
\hline Modelo de Page & $U=\exp \left(-k \cdot t^{n}\right)$ \\
Modelo de Henderson e Pabis & $U=a \cdot \exp (-k \cdot t)$ \\
Modelo de Lewis & $U=\exp (-k \cdot t)$ \\
Modelo de Midilli & $U=a \cdot \exp \left(-k \cdot t^{n}\right)+b \cdot t$ \\
\hline
\end{tabular}

Tabela 2: Indicadores de desempenho dos modelos matemáticos

\begin{tabular}{cc}
\hline Índice de desempenho & Equações \\
\hline Raiz do erro quadrático médio (REQM) & $R M S E=\sqrt{\frac{\sum(\text { obs }- \text { pred })^{2}}{n}}$ \\
Erro padrão de predição $(\%$ SEP) & $\% S E P=\frac{100}{\text { mean obs } \sqrt{\frac{\sum(\text { obs }- \text { pred })^{2}}{n}}}$ \\
Fator de viés $\left(\mathbf{B}_{\mathbf{f}}\right)$ & $B_{f}=10 \frac{\sum \log \left(\frac{\text { pred }}{n}\right)}{n b s}$ \\
Fator de precisão $\left(\mathbf{A}_{\mathbf{f}}\right)$ & $A_{f}=10 \frac{\Sigma\left|\log \left(\frac{\text { pred }}{n}\right)\right|}{n}$ \\
\hline
\end{tabular}

\section{RESULTADOS E DISCUSSÃO}

A Figura 1 representa graficamente os dados experimentais e os dados preditos pelo modelo. Foi observado que houve uma boa correlação entre tais valores para a mangaba pequena, com exceção do modelo de Midilli, que apresentou um comportamento linear. Por outro lado, para a mangaba grande, o modelo de Midilli foi que melhor se aproximou dos dados experimentais, de acordo com a Figura 1. 


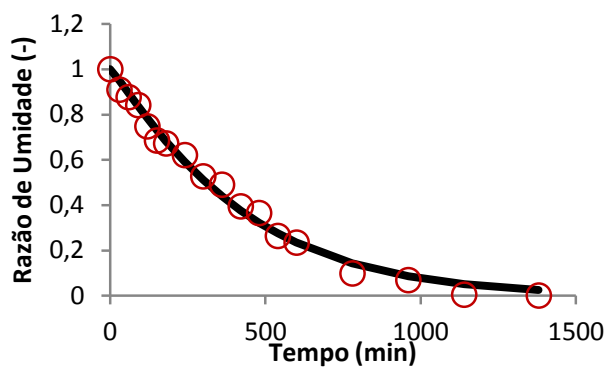

(a)

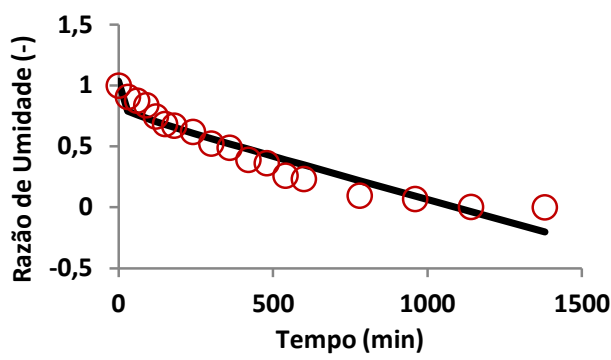

(c)

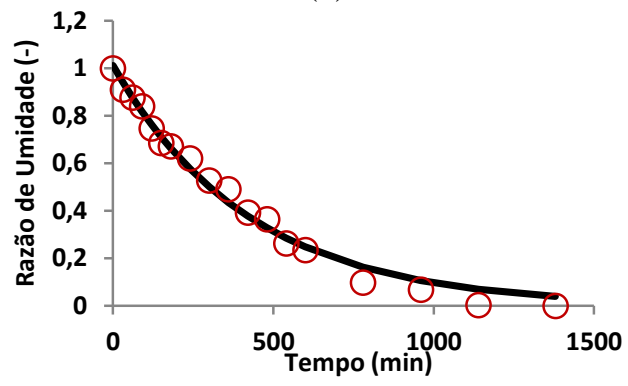

(e)

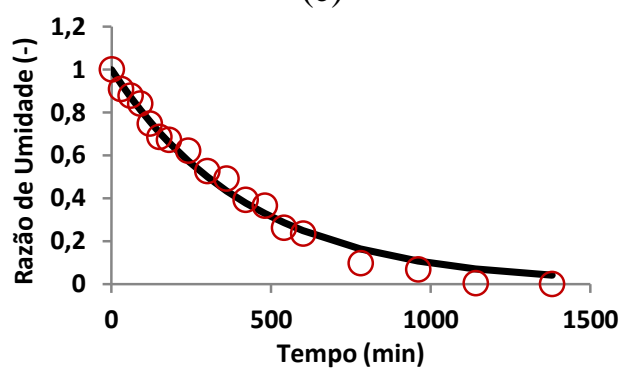

(g)

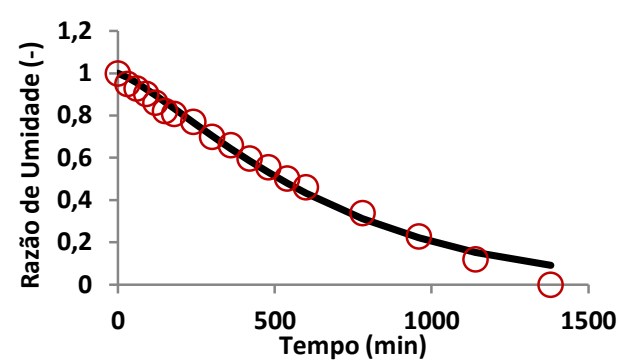

(b)

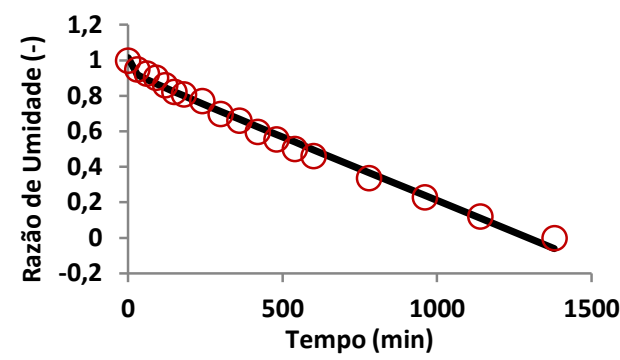

(d)

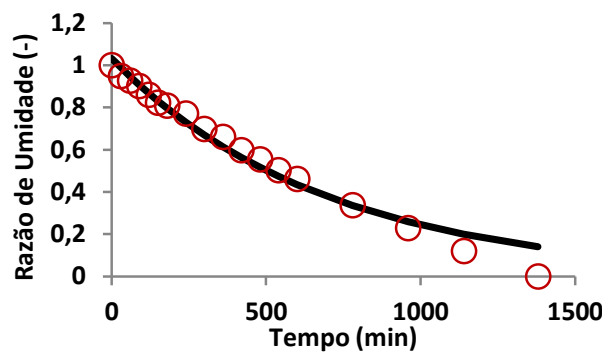

(f)

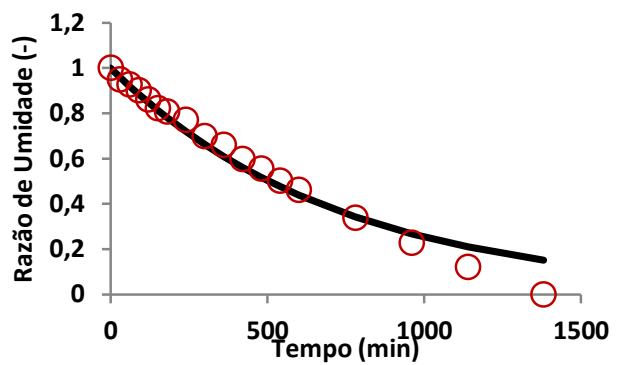

(h)

- Dados Experimentais

— Dados Preditos

Figura 1: Representação da cinética de secagem com os dados experimentais e preditos pelos modelos. (a) e (b) Modelo de Page; (c) e (d) Modelo de Midilli; (e) e (f) Modelo de Henderson e Pabis; $(g) e(h)$ Modelo de Lewis. (a), (c), (e) e (g) Cinéticas de secagem para as mangabas pequena e $(b),(d),(f) e(h)$

Cinéticas de secagem para as mangabas grandes.

Os parâmetros determinados pelos modelos estão dispostos na Tabela 3. Verifica-se que com relação ao coeficiente de secagem $(\mathrm{k})$, as mangabas com diâmetro maior tiverem valores, para essa constante, menores do que quando comparado com a mangaba pequena, isto provavelmente ocorreu devido às resistências encontradas pela água no momento da difusão, fator este proporcional ao volume da fruta, cujo valor foi 7,63117E-06 $\mathrm{m}^{3}$ e $4,97178 \mathrm{E}-05 \mathrm{~m}^{3}$ para a mangaba pequena e grande, respectivamente. Nota-se ainda que, para o modelo de Midilli, o coeficiente de secagem apresentou valor negativo para ambas as frutas, independente do 
diâmetro. Com relação ao parâmetro $\mathrm{n}$ do modelo de Page, observa-se que seus valores são maiores que 1, conforme Karathanos e Belessiotis, citado por Simal [6] estes autores também obtiveram este resultado com valores desta constante entre 1,02 e 1,79, dependendo do tipo de produto, conforme estes autores os valores de $\mathrm{n}$ aumentam com a existência de uma pele exterior do produto seco, as mangabas foram liofilizadas com a casca.

Tabela 3: Parâmetros determinados pelos modelos de secagem.

\begin{tabular}{|c|c|c|c|c|c|c|c|c|}
\hline \multirow{3}{*}{$\begin{array}{l}\text { Modelos } \\
\text { Matemátic } \\
\text { os }\end{array}$} & \multicolumn{8}{|c|}{ Parâmetros } \\
\hline & \multicolumn{4}{|c|}{ Mangaba Pequena } & \multicolumn{4}{|c|}{ Mangaba Grande } \\
\hline & $\mathrm{k}\left(\min ^{-1}\right)$ & $\mathrm{n}$ & $\mathrm{a}$ & $\mathrm{b}$ & $\mathrm{k}\left(\min ^{-1}\right)$ & $\mathrm{n}$ & $\mathrm{a}$ & $\mathrm{b}$ \\
\hline $\begin{array}{l}\text { Modelo de } \\
\text { Page }\end{array}$ & 0,0012 & 1,1106 & - & - & 0,0003 & 1,2569 & - & - \\
\hline $\begin{array}{l}\text { Modelo de } \\
\text { Midilli }\end{array}$ & $-3,5383$ & 0,0066 & 0,0255 & $\begin{array}{c}- \\
0,0007\end{array}$ & $-5,0146$ & 0,0015 & 0,0064 & $\begin{array}{c}- \\
0,0007\end{array}$ \\
\hline $\begin{array}{l}\text { Modelo de } \\
\text { Lewis }\end{array}$ & 0,0023 & - & - & - & 0,0014 & - & - & - \\
\hline
\end{tabular}

A Tabela 4 apresenta os resultados dos índices de desempenho que foram considerados. $\mathrm{O}$ REQM faz a comparação entre os valores experimentais e os preditos, o modelo ideal será aquele que apresentar um valor reduzido deste parâmetro, o menor valor obtido foi no modelo de Page para ambas as frutas, independente do diâmetro, o modelo de Midilli e o de Lewis apresentaram maior divergência entre os dados experimentais e preditos para a mangaba pequena e grande, respectivamente. O fator de precisão estabelece a diferença média entre os dados experimentais e preditos, seu aumento sugere a baixa capacidade da precisão entre os valores estimados e observados. Contudo, o modelo adequado será aquele em que este fator for menor, no caso, o modelo de Page e de Midilli para a mangaba pequena e grande, respectivamente.

$\mathrm{O}$ erro padrão de predição, \%SEP, faz análise do residual dos modelos, em razão disto o seu valor deve ser reduzido, com isto, verifica-se que o Modelo de Page se ajustou novamente aos dados experimentais tanto para a mangaba pequena, quanto para a grande. Finalmente o fator de viés, $\mathrm{B}_{\mathrm{f}}$, é o parâmetro que indica a melhor performance do modelo. De acordo com Ross [9], a concordância perfeita entre os valores preditos e os observados é representada por um $\mathrm{B}_{\mathrm{f}}$ igual a 1. Dentre os modelos utilizados, o de Midilli e o de Page foram os que mais se aproximaram de 1, para a mangaba grande, no entanto para a mangaba pequena, o Modelo de Page apresentou um menor valor de $\mathrm{B}_{\mathrm{f}}$, já o de Midilli foi o que mais divergiu.

Tabela 4: Resultados dos indicadores de desempenho.

\begin{tabular}{|c|c|c|c|c|c|c|c|c|}
\hline \multirow{3}{*}{$\begin{array}{c}\text { Modelos } \\
\text { Matemático } \\
\text { S }\end{array}$} & \multicolumn{8}{|c|}{ Parâmetros } \\
\hline & \multicolumn{4}{|c|}{ Mangaba Pequena } & \multicolumn{4}{|c|}{ Mangaba Grande } \\
\hline & $\begin{array}{l}\text { RMS } \\
\text { E }\end{array}$ & $\mathbf{A}_{\mathbf{f}}$ & $\mathbf{B}_{\mathrm{f}}$ & \%SEP & $\begin{array}{l}\text { RMS } \\
\text { E }\end{array}$ & $\mathbf{A}_{\mathbf{f}}$ & $\mathbf{B}_{\mathrm{f}}$ & $\%$ SEP \\
\hline Page & 0,0275 & 1,9198 & 1,8446 & 5,6298 & 0,0221 & 1,7334 & 1,6735 & 3,5545 \\
\hline Midilli & 0,0752 & 2,3143 & 2,1829 & 15,3811 & 0,0225 & 1,6806 & 1,6372 & 3,6147 \\
\hline Lewis & 0,0287 & 2,0560 & 1,9563 & 5,8801 & 0,0259 & 1,8163 & 1,7294 & 4,1568 \\
\hline $\begin{array}{c}\text { Henderson e } \\
\text { Pabis }\end{array}$ & 0,0289 & 2,0434 & 1,9476 & 5,9098 & 0,0253 & 1,8299 & 1,7281 & 4,0707 \\
\hline
\end{tabular}

Perante os resultados, deduz-se que o Modelo de Page é o que mais se ajusta aos dados experimentais independentemente do raio da mangaba. Tal circunstancia foi observado por Marques [5] na liofilização de polpas de abacaxi, goiaba e manga. Conforme o estudo de Gunhan 
et al. [10] com secagem de folhas de louro com três diferentes umidades relativas $(5 \%, 15 \%$ e $25 \%)$ e temperaturas $\left(40,50\right.$ e $\left.60{ }^{\circ} \mathrm{C}\right)$ e uma velocidade do ar constante de $1,5 \mathrm{~m} / \mathrm{s}$, o modelo de Page também forneceu um bom ajuste com os valores observados, juntamente com outros modelos como por exemplo o modelo de Midilli, no entanto o modelo de Page foi selecionado devido a sua simplicidade. Tunde-Akintunde [11] realizou uma secagem solar com pimenta malagueta e observou que o modelo de Page descreveu melhor as características desta secagem.

\section{CONCLUSÃO}

Neste trabalho o modelo matemático de secagem que mais se adequou a liofilização de mangabas grandes e pequenas foi avaliado. Observou-se que dentre os modelos analisados, o modelo de Page apresentou cinéticas cujos dados preditos se correlacionaram bem com os valores observados tanto para a mangaba grande quanto para pequena. Esta escolha foi realizada pelos indicadores de desempenho, onde o modelo de Page ofereceu resultados mais precisos quando comparado com os demais modelos, apresentando os seguintes resultados para os indicadores: RMSE: 0,0275, $\mathrm{A}_{\mathrm{f}}: 1,9198, \mathrm{~B}_{\mathrm{f}}: 1,8446$ e \%SEP: 5,6298 para a mangaba pequena e RMSE: 0,0221, $\mathrm{A}_{\mathrm{f}}: 1,7334, \mathrm{~B}_{\mathrm{f}}: 1,6735$ e \%SEP: 3,5545 para a mangaba grande. Com relação aos parâmetros encontrados, a constante $\mathrm{k}$ foi menor para as mangabas grandes, provavelmente devido a seu volume e, quanto a constante $\mathrm{n}$, os seus valores foram maiores que 1 , apresentando os seguinte resultados 1,1106 e 1,2569 para a mangaba pequena e grande, respectivamente.

\section{AGRADECIMENTOS}

À Universidade Federal de Sergipe pela infraestrutura oferecida.

\section{REFERÊNCIAS BIBLIOGRÁFICAS}

1. Sampaio TS, Nogueira PCL. Volatile components of mangaba fruit (Hancornia speciosa Gomes) at three stages of maturity. Food Chem. 2006 Apr;95(4):606-610, doi: 10.1016/j.foodchem.2005.01.038

2. Lima JP, Fante CA, Pires CRF, Nunes EE, Alves RR, Elias HHS, Nunes CA, Boas EVBV. The antioxidative potential and volatile constituents of mangaba fruit over the storage period. Sci Hortic. 2015 Oct;194:1-6, doi: 10.1016/j.scientia.2015.05.035

3. Marques LG, Prado MM, Freire JT. Rehydration characteristics of freeze-dried tropical fruits. Food Sci and Technol. 2009 Sep;42(7):1232-1237, doi:10.1016/j.lwt.2009.02.012

4. Shofian NM, Hamid AA, Osman A, Saari N, Anwar F, Dek MSP, Hairuddin MR. Effect of freezedrying on the antioxidant compounds and antioxidant activity of selected tropical fruits. Int J Mol Sci. 2011 Jul;12(7):4678-4692, doi: 10.3390/ijms12074678

5. Marques LG. Liofilização de frutas tropicais. [Tese]. São Carlos (SP): Universidade Federal de São Carlos; 2008. 255 p.

6. Freire JT, Ferreira MC. Aplicações Tecnológicas em Sistemas Particulados. São Paulo: Editora Animeres, 2009.

7. Dinani ST, Hamdami N, Shahedi M, Havet M. Mathematical modeling of hot air/electrohydrodynamic (EHD) drying kinetics of mushroom slices. Energ Convers Manage. 2014 May;86: 70-80, doi: /10.1016/j.enconman.2014.05.010

8. Aregbesola AO, Ogunsina BS, Sofolahan AE, Chime NN. Mathematical modeling of thin layer drying characteristics of dika (Irvingia gabonensis) nuts and kernels. Niger Food J. 2015 May;33:83-89, doi: 10.1016/j.nifoj.2015.04.012

9. Ross T. Índices for performance evaluation of predictive models in food microbiology. J Appl Bacteriol. 1996 Mar;81:501-508, doi: 10.1111/j.1365-2672.1996.tb03539.x

10. Gunhan T, Demir V, Hancioglu E, Hepbasli A. Mathematical modeling of drying of bay leaves. Energ Convers manage. 2005 Jul;46(11-12): 1667-1679, doi: 10.1016/j.enconman.2004.10.001

11. Tunde-akintunde TY. Mathematical modeling of sun and solar drying of chilli pepper. Renew Energ. $2011 \mathrm{Jul} ; 36$ (8): 2139-2145, doi: 10.1016/j.renene.2011.01.017 